\title{
Primary cutaneous umbilical melanoma
}

\section{Dear Editor,}

A 59-year-old woman with a pre-existing asymptomatic pigmented nevus on the umbilicus for the past 20 years was seen in the outpatient dermatology clinic for a 2-week history of a raised, bleeding pigmented papule overlying the nevus. Physical examination showed a $7 \times 7 \mathrm{~mm}$ ulcerated papule overlying a $2.2 \times 1.8 \mathrm{~cm}$ darkly pigmented patch over the umbilicus, and dermoscopy showed an asymmetrical, homogeneously hyperpigmented ulcerated papule atop a similarly hyperpigmented lesion with irregular borders (Fig. 1). Biopsy showed pigment-containing atypical nevus cells arranged in nests and distributed in a lentiginous fashion along the dermal-epidermal interface with Pagetoid spread in the upper epidermis (Fig. 2). Physical examination did not demonstrate palpable inguinal lymph nodes or other features to suggest distant metastasis. Additionally, initial pre-operative positron emission tomographycomputed tomography scan for staging did not reveal any distant metastasis. Wide excision of the lesion with $2 \mathrm{~cm}$ margins and down to the base of the cicatrix showed an ulcerated superficial spreading melanoma with Clark level IV and Breslow's thickness of $6.4 \mathrm{~mm}$, and mitotic rate of $11 / \mathrm{mm}^{2}$. Tumour-infiltrating lymphocytes and microsatellites were present, without perineural and lymphovascular invasion. Sentinel lymph node biopsy from bilateral inguinal lymph nodes were positive. Complete nodal dissection was not performed, since it has been shown not to improve survival outcomes over observation alone. ${ }^{1}$ The tumour was staged to be pT4b $(>4.0 \mathrm{~mm}$ thickness, with ulceration) and N3c ( $>1$ tumour-involved node, with microsatellite metastases), as per the Tumor, Node, Metastasis (TNM) system, American Joint Committee on Cancer (AJCC) 8th edition. ${ }^{2}$ Further testing showed that the tumour tissue was wild type for both BRAF and c-kit (exons 9/11/13/17) and hence the patient was started on adjuvant immunotherapy with Nivolumab by the oncologist. However, the disease continued to progress and interval positron emission tomography and computed tomography scan done showed distant metastases to the rib, residual unresected left inguinal node and upper lobe of the right lung.

Discussion. A retrospective review of 48 patients with primary cutaneous melanoma in Singapore between 1998 and 2008 found $44 \%$ of the cases were located on the palms and soles (21), and only $19 \%$ were located on

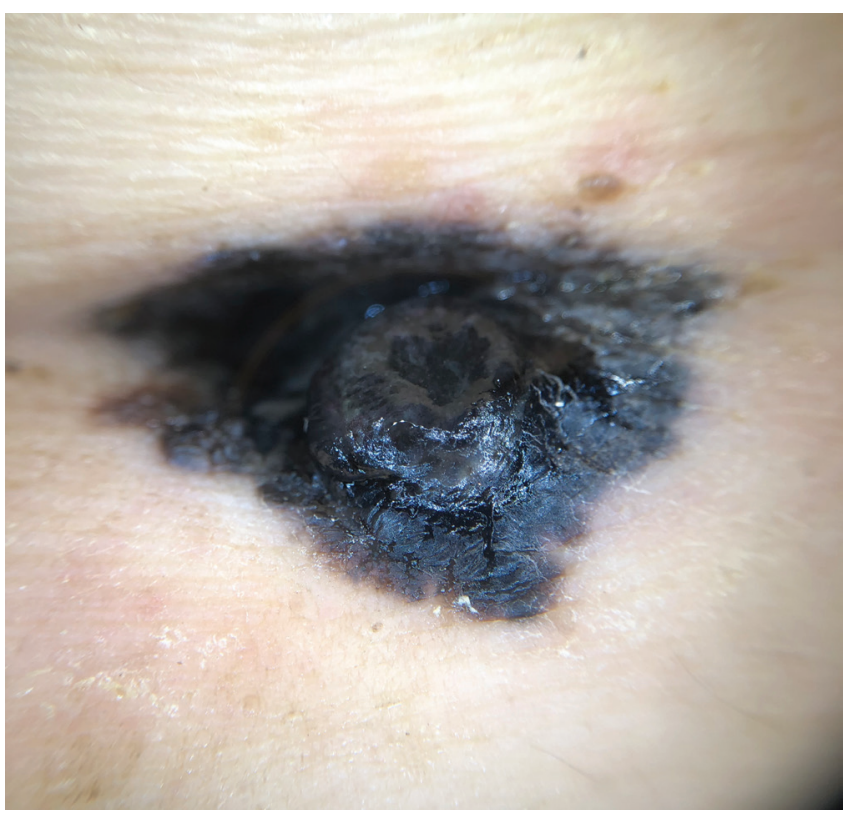

Fig. 1. Dermoscopy showing an asymmetrical, homogeneously hyperpigmented ulcerated papule atop a similarly hyperpigmented lesion with irregular borders.

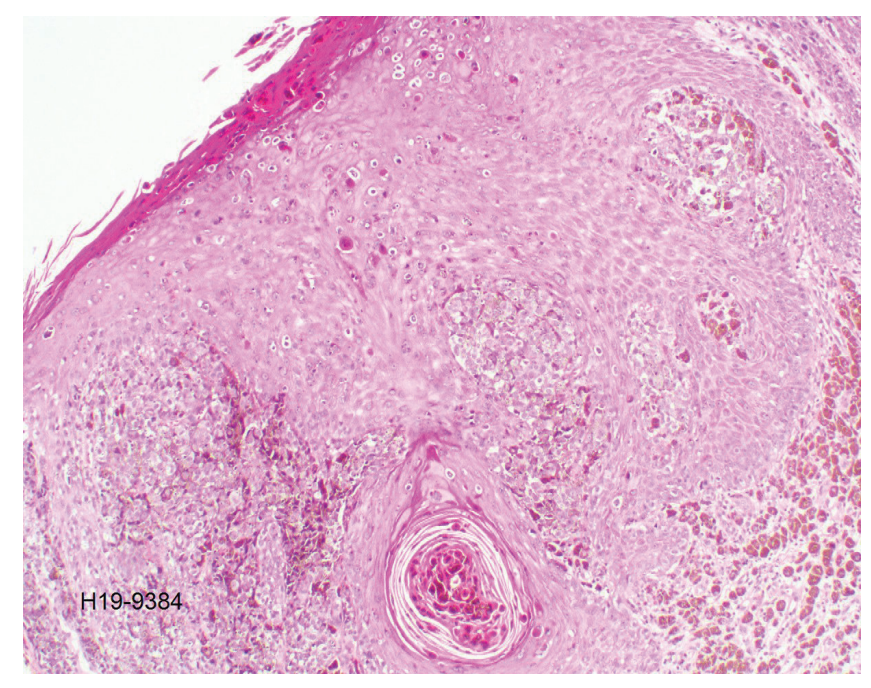

Fig. 2. Histology showing atypical nevus cells arranged in nests and along the dermal-epidermal interface with Pagetoid spread in the upper epidermis.

the trunk (9), ${ }^{3}$ although it was not specified if any of the cases were located in the umbilicus. Primary cutaneous umbilical melanoma is rare, with only 46 cases reported in the literature worldwide. Nearly two-thirds of the cases were women (31/46), and the average age of the 
patients was 52 years (range 21-84 years). Sunbathing practices in females and the potential photoprotective effects of terminal hairs around the periumbilical area in males have been suggested as possible explanations for the female predilection. ${ }^{4}$

This rare case highlights several learning points. Firstly, lesions in the umbilicus may be concealed in the concavity of the umbilicus or dismissed as benign. Benign conditions include keratoses, nevi or even omphalolith, while malignant conditions may include basal cell carcinoma or cutaneous metastases, better known as Sister Mary Joseph's nodules. Secondly, patients should be advised to perform periodic selfexamination of umbilical lesions. Any change in appearance or symptoms (asymmetry, rapid increase in size, colour change, bleeding, pruritus) should prompt an early excision biopsy with adequate consideration of the unique anatomy of the region. ${ }^{4}$ Thirdly, as the umbilicus is a concaved or flexural site, histological samples from such special sites should be interpreted by an experienced pathologist to avoid over-diagnosis of melanoma and over-aggressive treatment, which may include extensive surgery and adjuvant immunotherapy. This is because nevi located at flexural sites (including the umbilicus) can show a nested and dyshesive pattern histological appearance, with enlarged junctional nests and diminished cohesion of melanocytes, resembling that of a melanoma. ${ }^{5}$ Lastly, the potential importance of sentinel lymph node biopsy in the management of umbilical melanoma. The umbilical cord circulatory structures undergo fibrosis at birth and persist as ligaments that remain connected to intra-abdominal organs, including the liver, urinary bladder and the superior vesicle arteries. ${ }^{6}$ These vestigial remnants and their connections have been postulated to serve as potential metastatic pathways for distant spread to intra-peritoneal structures and may also increase the risk of incomplete clearance or tumour recurrence. ${ }^{7}$ As such, the role of sentinel lymph node biopsy has been questioned. However, similar to the series of patients in a study by Charles et al., ${ }^{4}$ who reported the first 2 cases of positive sentinel lymph node biopsies in the setting of primary umbilical melanoma, sentinel lymph node biopsy from the inguinal basins in our patient was also positive, suggesting the importance of lymphatic spread and the utility of sentinel lymph node biopsies in umbilical melanoma, despite the variable lymphatic drainage pattern of the umbilicus.

\section{Acknowledgment}

The authors would like to thank Dr Sim Chee Seng for his invaluable help with interpreting the histology results and providing the histology photographs.

\section{REFERENCES}

1. Faries MB, Thompson JF, Cochran AJ, et al. Completion Dissection or Observation for Sentinel-Node Metastasis in Melanoma. N Engl J Med 2017;376:2211-22.

2. Gershenwald JE, Scolyer RA. Melanoma Staging: American Joint Committee on Cancer (AJCC) 8th Edition and Beyond. Ann Surg Oncol 2018;25:2105-10

3. Lee HY, Chay WY, Tang MB, et al. Melanoma: differences between Asian and Caucasian patients. Ann Acad Med Singap 2012;41:17-20.

4. Charles KB, Chan MP, Smith NR, et al. Primary Cutaneous Umbilical Melanoma: The Michigan Experience. Dermatol Surg 2020;46:312-8.

5. Mason AR, Mohr KR, Koch LH, et al. Nevi of special sites. Clin Lab Med 2011;31:229-42.

6. Hegazy AA. Anatomy and embryology of umbilicus in newborns: a review and clinical correlations. Front Med 2016;10:271-7.

7. Cecchi R, Pavesi M, Buralli L, et al. Primary umbilical melanoma. Australas J Dermatol 2009;50:220-2.

Ki Wei Tan, ${ }^{1}$ FAMS (Dermatology), Jason Yongsheng Chan, ${ }^{2,3}$ FAMS (Medical Oncology)

\footnotetext{
${ }^{1}$ Department of Dermatology, Changi General Hospital, Singapore

${ }^{2}$ Division of Medical Oncology, National Cancer Centre Singapore, Singapore

${ }^{3}$ Oncology Academic Clinical Program, Duke-NUS Medical School, Singapore
}

Correspondence: Dr Ki Wei Tan, Department of Dermatology, Changi General Hospital, 2 Simei Street 3, Singapore 529889.

Email: tan.ki.wei@singhealth.com.sg 